08,11

\title{
Расчет динамики границы аморфная фаза-кристалл при твердофазной взрывной кристаллизации
}

\author{
(C) А.А. Чеврычкина, Н.М. Бессонов, А.Л. Корженевский \\ Институт проблем машиноведения РАН, \\ Санкт-Петербург, Россия \\ E-mail: alekorzh@mail.ru
}

Поступила в Редакцию 5 июля 2021 г. В окончательной редакции 10 июля 2021 г.

Принята к публикации 10 июля 2021 г.

Выведено нелинейное дифференциальное уравнение, позволяющее описывать динамику фронта твердофазной взрывной кристаллизации в существенно более широкой области значений параметров по сравнению с имеющимися в литературе теоретическими результатами. Детально изучены численно особенности перехода автоколебательной моды движения фронта в режим его самораспространения с постоянной скоростью.

Ключевые слова: взрывная кристаллизация, автоколебания межфазной границы стекло-кристалл, самораспространяющийся фронт.

DOI: 10.21883/FTT.2021.11.51599.162

\section{1. Введение}

Динамика взрывной кристаллизации (ВК) занимает особое место в кинетике различных фронтов физической или химической природы, таких как грани роста кристаллов, межфазные границы при фазовых переходах, магнитные или сегнетоэлектрические доменные стенки и т.п. Важность исследования ВК при кристаллизации аморфной фазы обусловлена тем, что лежащая в основе этого процесса положительная обратная связь между выделением скрытой теплоты и скоростью фронта играет ключевую роль и во многих других, более сложных для количественного описания явлений, например, автокаталитических экзотермических химических реакций или взаимосвязи конкурирующих фазовых переходов при самораспространяющемся высокоскоростном синтезе [1-3]. Вместе с тем, несмотря на качественную схожесть с некоторыми другими диффузионноконтролируемыми процессами, например, быстрым направленным затвердеванием расплавов сплавов, более детальный анализ ВК указывает и на отличия в их математическом описании.

В ранних теоретических работах анализ ВК проводился в рамках предположения о постоянной скорости фронта [4-6], см. также обзор [7]. Было показано, что для самораспространяющихся фронтов ВК их скорость определяется условием теплового баланса, которое при определенных значениях параметров допускает не единственность решения [5-7]. Обнаружение этого обстоятельства позволило предсказать возможность теплового гистерезиса не только в приближении постоянной скорости фронта, но и в более общих случаях, однако, принципиально ограниченных требованием квазистационарности режима, см., например, [8].

Экспериментально ВК наблюдалась в пленках целого ряда чистых элементов и химических соединений, относящихся к материалам различных классов. При этом были выделены типичные черты явления, наблюдаемые на достаточно больших пространственных масштабах и временах: пороговый характер возникновения и подавления ВК, ее зависимость от температуры подложки, толщины аморфной пленки, способа ее приготовления, тепловых свойств материала подложки и т. п. Было установлено, что в зависимости от экспериментальных условий, ВК может проходить как в сохраняющем твердость материале, так и с образованием промежуточной жидкой фазы. Кроме того, кинетика процесса может сопровождаться зарождением множества кристаллитов в аморфной матрице или реализовываться распространением одиночного фронта стекло-кристалл, см., например, [9-15].

Ocобое внимание привлекли эксперименты, в которых post mortem наблюдались периодические изменения толщин аморфных пленок и характерных размеров зерен в поликристаллических продуктах ВК [16]. Для объяснения этих эффектов было высказано предположение, что они являются следствием периодических осцилляций скорости фронта [6]. Возможность возникновения таких осцилляций была продемонстрирована в рамках анализа линейной устойчивости равномерного движения плоских фронтов [6,17-19]. В последующих теоретических работах был изучен и их нелинейный режим движения как для самораспространяющихся фронтов ВК, так и в условиях слабой поддержки их движения подвижным тепловым источником (обычно сканирующим лазерным лучом $[17,20,21])$. Необходимо, однако, отметить, что использованные методы вывода формул достаточно сложны, сами полученные формулы весьма громоздки и к тому же применимы лишь в очень узких областях значений параметров, что затрудняет и сильно ограничивает их практическое применение. В свою очередь, численные расчеты, выполненные в рамках получившего широкое распространение метода фазового поля, см., например, [22-24] или методом 
молекулярной динамики [25-27], эффективны лишь для описания особенностей ВК в моделях конкретных стекол, но не позволяют делать предсказаний обобщающего характера.

В последние годы произошел значительный прогресс в экспериментальных возможностях наблюдения in situ процессов, сопровождающих ВК. Он связан в первую очередь с разработкой новой методики (dynamic transmission electron microscopy), с помощью которой удалось добиться гораздо более высокого пространственного и временного разрешения структуры кристаллизующегося материала [28-30]. В частности, проведенные в [28-30] замечательные эксперименты показали, что переход аморфного состояния в кристалл Ge происходит по механизму бокового, а не рассмотренного в теоретических работах [5,6,17-21] нормального роста. T.е. сначала на фронте возникает локальный закристаллизовавшийся выступ, который затем расширяется и охватывает всю поверхность фронта. При этом выступов может быть несколько, расширяются они независимым образом, что ясно указывает на нуклеационный характер процесса.

В целом можно сказать, что в настоящее время накопленный разнообразный экспериментальный материал по ВК намного превосходит имеющиеся теоретические результаты. Ощущается острая необходимость в разработке новых методов для проведения количественных теоретических расчетов как в рамках традиционных общих макрокинетических моделей с простой геометрией фронта ВК, так и для моделей, оперирующих на наномасштабах.

Цель настоящей статьи состоит в том, чтобы в рамках традиционного феноменологического подхода предложить новый теоретический метод расчета динамики фронта ВК, одновременно более простой и в то же время позволяющий описывать ее в гораздо более широкой области параметров, чем это было сделано в [17,20,21]. При этом основное внимание будет уделено расчету автоколебательной динамики фронта и ее трансформации в самоподдерживающийся режим.

\section{2. Формулировка модели и исходные уравнения}

В базовой макрокинетической модели фронт ВК рассматривается как бесконечно тонкий тепловой источник, зависимость скорости которого от его температуры содержит экспоненциальный аррениусовский фактор. Процесс теплового обмена в объеме материала описывается стандартным уравнением теплопроводности, а сток тепла из пленки в подложку рассматривается в релаксационном или диффузионном режиме [7,31]. Поскольку в последние годы растет интерес к ВК во все более тонких аморфных пленках, в нашей статье мы рассмотрим первый из этих режимов. В этом случае тепловую задачу можно решать в приближении одномерной модели, закон стока тепла в которой в простейшем случае считается ньютоновским. Если температура подложки $T_{S}$ недостаточно высока, и процесс ВК реализуется при поддержке сканирующего лазерного луча, то уравнение теплопроводности содержит дополнительный источник. В результате уравнения модели приобретают вид

$$
\begin{gathered}
\frac{\partial T}{\partial t}=D \frac{\partial T}{\partial z^{2}}-\Gamma\left(T-T_{S}\right)+J, \\
J(z, t)=q_{0} V \delta(z-Z(t))+P\left(z-V_{L} t\right), \\
V\left(T_{i}\right)=V_{0} \exp \left(-\frac{E}{T_{i}}\right),
\end{gathered}
$$

где $D-$ коэффициент температуропроводности, коэффициент $\Gamma$ задает скорость стока тепла в подложку, $q_{0}=L / c, L-$ теплота кристаллизации, $c-$ теплоемкость, $V=\dot{Z}$ - скорость фронта, $Z(t)$ - его координата, $\delta(z-Z(t))$ - дельта-функция Дирака, функция $P\left(z-V_{L} t\right)$ описывает тепловой вклад от лазерной подсветки, $V_{L}$ - заданная скорость лазерного луча, $T_{i}$ - температура фронта, $V_{0}-$ феноменологический параметр, $E$ - эффективная энергия активации.

\section{3. Вывод уравнения движения фронта в дифференциальной форме}

Динамика координаты фронта $Z(t)$ определяется из условия самосогласованного равенства температуры фронта $T_{i}(V)$, взятой из формулы (3), переписанной в виде

$$
T_{i}(V)=-\frac{E}{\ln \left(\frac{V}{V_{0}}\right)}
$$

и найденной из решения уравнения (1). В силу линейности этого уравнения его решение можно записать в виде свертки функции Грина $G(z, t)$ с источником $J(z, t)$ в $(2)$. При этом для получения результатов в аналитическом виде удобно записать функцию Грина $G(z, t)$ через ее пространственный Фурье-образ

$$
G(z, t)=\int_{-\infty}^{+\infty} \frac{d k}{2 \pi} \exp \left[\left(-D k^{2}-\Gamma\right) t+i k z\right]
$$

затем подставить (5) в выражение для решения (1) на фронте в экспериментально существенном, установившемся при больших временах, режиме

$$
\begin{gathered}
T(Z(t), t)=T_{S}+I_{1}+I_{2}, \\
I_{1}=q_{0} \int_{-\infty}^{t} d t^{\prime} \int_{-\infty}^{+\infty} d z^{\prime} G\left(Z(t)-z^{\prime}, t-t^{\prime}\right) V\left(t^{\prime}\right) \delta\left(z^{\prime}-Z\left(t^{\prime}\right)\right), \\
\left.I_{2}=\int_{-\infty}^{t} d t^{\prime} \int_{-\infty}^{+\infty} d z^{\prime} G\left(Z(t)-z^{\prime}, t-t^{\prime}\right) P\left(z^{\prime}-V_{L} t^{\prime}\right)\right) .
\end{gathered}
$$

Вклады $I_{1}$ и $I_{2}$ в уравнении (6), обусловленные выделением теплоты кристаллизации и лазерной подсветкой, 
целесообразно рассчитать по отдельности. Первый из них можно представить в виде следующего ряда:

$$
\begin{aligned}
I_{1} & =q_{0} \int_{-\infty}^{t} d t^{\prime} V\left(t^{\prime}\right) G\left(Z(t)-Z\left(t^{\prime}\right)\right) \\
& =q_{0} \int_{-\infty}^{t} d t^{\prime}\left[V(t)+\dot{V}(t)\left(t^{\prime}-t\right)+\ldots\right] \\
& \times \int_{-\infty}^{+\infty} d k\left[1-i k \frac{\dot{V}(t)}{2}\left(t^{\prime}-t\right)^{2}-i k \frac{\ddot{V}(t)}{6}\left(t^{\prime}-t\right)^{3}+\ldots\right] \\
& \times \exp \left[D k^{2}-i k V(t)\right]\left(t^{\prime}-t\right) .
\end{aligned}
$$

Поскольку скорость $V(t)$ и ее производные входят в (7) только как параметры, можно в явном виде выполнить интегрирование по времени $t^{\prime}$ во всех членах ряда несмотря на то, что динамика фронта пока не определена. Затем интегралы по $k$ элементарно берутся по вычетам, а результат удобно записать в компактном виде

$$
\begin{aligned}
I_{1} & =q_{0} \sqrt{B} \\
& \times\left\{1-\beta\left(1-\frac{3}{2} \beta\right) \ddot{Z}(t)+\beta^{2}\left(\frac{3}{2}-\frac{5}{2} \beta\right) \dddot{Z}(t)+\ldots\right\},
\end{aligned}
$$

где функция $\beta(V)$ определена как

$$
\beta(V) \equiv \frac{V^{2}}{V^{2}+4 \Gamma D}, \quad \beta_{L} \equiv \beta\left(V=V_{L}\right)
$$

Если скорость перемещения лазерного луча $V_{L}$ постоянна, то вклад подсветки $I_{2}$ в значение $T_{i}$ можно записать в виде свертки источника $P\left(z-V_{L} t\right)$ с функцией Грина $G_{s}$ стационарного уравнения теплопроводности

$$
\begin{gathered}
I_{2}=\int_{-\infty}^{+\infty} G_{S}\left(Z(t)-z^{\prime}\right) P\left(z^{\prime}\right) d z^{\prime}, \\
G_{S}(Z)=\frac{\sqrt{\beta_{L}}}{V_{L}} \exp \left[-\frac{V_{L}\left(z+\beta_{L}^{-\frac{1}{2}}|z|\right)}{2 D}\right],
\end{gathered}
$$

В случае когда фронт кристаллизации находится вне лазерного пятна, этот вклад экспоненциально спадает с увеличением расстояния фронта от пятна.

Приравняв значения температуры на фронте $T_{i}$ в (4) и (6), с учетом (8) и (10) получаем уравнение, описывающее динамику координаты фронта $Z(t)$ в форме нелинейного дифференциального уравнения бесконечного порядка

$$
\begin{aligned}
T_{S} & +q_{0} \sqrt{\beta}\left\{1-\beta\left(1-\frac{3}{2} \beta\right) \ddot{Z}(t)+\beta^{2}\left(\frac{3}{2}-\frac{5}{2} \beta\right) \dddot{Z}(t)+\ldots\right\} \\
& +I_{2}\left(Z_{S}\right) \exp \left[-\frac{V_{L}\left(Z-Z_{S}\right)}{2 D l}\right]=-\frac{E}{\ln \left(\frac{V}{V_{0}}\right)}
\end{aligned}
$$

где длина $l$ определена равенством $l^{-1}\left(V_{L}\right) \equiv 1+\beta_{L}^{-\frac{1}{2}}$.
В уравнении (12) $Z_{s}=V_{L} t+Z_{0}$, где $Z_{0}$ расстояние между лазерным пятном и положением фронта в случае его равномерного движения со скоростью $V_{L}$ Соответственно, значение $Z_{0}$ определяется условием

$$
T_{s}+q_{0} \sqrt{\beta_{L}}+I_{2}\left(Z_{s}\right)=-\frac{E}{\ln \left(\frac{V_{L}}{V_{0}}\right)} .
$$

Преимущества записи левой части уравнения (12) в дифференциальной форме по сравнению с интегралом в (6) заключаются в возможности построения приближенных решений (12) в аналитическом виде и их ясной физической интерпретации. Этим вопросам посвящен следующий параграф.

\section{4. Стационарные и автоколебательные режимы динамики фронта}

Очевидно, что скорость фронта в стационарных режимах, реализация которых требует лазерной поддержки, равна $V_{L}$. В случае самораспространяющихся фронтов $\mathrm{BK}$, она определяется устойчивым решением $V_{s}$ уравнения (13), в котором опущен член $I_{2}$. Анализировать общие свойства уравнений (12), (13), содержащих несколько размерных параметров, удобнее, если перейти к безразмерным переменным. Поэтому введем новые время и длину как

$$
t \rightarrow \frac{2 D}{V_{L}^{2}} t, \quad Z \rightarrow \frac{2 D}{V_{L}} Z
$$

Помимо введенного выше параметра $\beta$, будем использовать еще два безразмерных параметра, $\alpha$ и $R$, которые задаются соотношениями

$$
\alpha \equiv \frac{q_{0}}{E} \sqrt{\beta_{L}} \ln ^{2}\left(\frac{V_{L}}{V_{0}}\right), \quad R \equiv \frac{I_{2}}{q_{0} l \sqrt{\beta_{L}}} .
$$

Учитывая, что даже в том случае, когда уравнение (13) имеет решение с постоянной скоростью, оно может оказаться неустойчивым, введем также безразмерное отклонение координаты фронта от ее значения в стационарном режиме $h(t)=Z(t)-Z_{s}$.

Запишем теперь разность уравнений (12) и (13) во введенных безразмерных переменных и параметрах

$$
\sum_{n=2}^{\infty} M_{n} \frac{d^{n} h}{d t^{n}}+M_{1} \dddot{h}+M_{0} \ddot{h}+F(\dot{h})+g\left[\exp \left(-\frac{h}{l}\right)-1\right]=0
$$

где

$$
\begin{aligned}
& M_{1}(\dot{h})=\frac{\beta^{\frac{5}{2}}(\dot{h})}{(\dot{h}+1)^{6}}\left(\frac{3}{2}-\frac{5}{2} \beta(\dot{h})\right), \\
& M_{0}(\dot{h})=\frac{\beta^{\frac{3}{2}}(\dot{h})}{(\dot{h}+1)^{3}}\left(\frac{3}{2} \beta(\dot{h})-1\right),
\end{aligned}
$$




$$
\begin{gathered}
F=\sqrt{\beta(\dot{h})}-\sqrt{\beta_{L}}\left[1+\frac{1}{\alpha} \frac{\ln \left(\frac{V_{L}}{V_{0}}\right) \ln (\dot{h}+1)}{\ln \left(\frac{V_{L}}{V_{0}}\right)+\ln (\dot{h}+1)}\right], \\
g=R l \sqrt{\beta_{L}}, \\
\beta(\dot{h}) \equiv \frac{(1+\dot{h})^{2}}{(1+\dot{h})^{2}+\left(\frac{1}{\beta_{L}}-1\right)} .
\end{gathered}
$$

Гипермассы $M_{n}$ при $n>1$ можно вычислить аналогично тому, как это было сделано для $M_{0}$ и $M_{1}$.

Заметим, что полезно интерпретировать уравнение (16) как выполнение баланса сил, включающих гиперинерционные (возникающие в силу запаздывания, как в классической постановке задачи о самоторможении неточечного модельного электрона), инерционную силу, силу нелинейного вязкого трения и возвращающую силу.

Покажем теперь, что при малых значениях безразмерного параметра $g$ (т.е. при достаточности слабой лазерной поддержке движения фронта) автоколебательные решения с хорошей точностью могут быть найдены из решения уравнения для нелинейного осциллятора

$$
M_{0}(\grave{h}) \ddot{h}+F(\dot{h})+g[\exp (-h / l)-1]=0 .
$$

Из (18) ясно, что вообще какие-либо колебательные решения уравнения (22) существуют только при условии $\beta<2 / 3$ (т.е. при наличии достаточно большого теплоотвода в подложку, что характерно для очень тонких пленок), когда знак $M_{0}$ совпадает со знаком возвращающей силы. Отметим, что в отличие от схожей задачи быстрой направленной кристаллизации (БНК) сплава [32-34], эта „возвращающая“ сила в (22) отрицательна, что является следствием присущей процессу ВК положительной обратной связи из-за другого по сравнению с БНК знака градиента температуры.

Будут ли колебательные решения (22) автоколебательными, зависит при $g \ll 1$ от знака производной „силы трения“ $F$ (взятой при $\left.V=V_{L}\right)$. Из (19) находим на плоскости $\alpha-\beta$ линию смены знака $d F / d \dot{h}$ (при $\left.V=V_{L}\right)$ :

$$
\frac{1}{2} \frac{\beta^{\prime}}{\beta} V_{L}-\frac{1}{\alpha}=0
$$

или, эквивалентно,

$$
\beta=\frac{\alpha-1}{\alpha} .
$$

Эта линия с точностью до $O(g)$ совпадает с линией бифуркации Хопфа стационарного движения фронта

$$
\beta=\frac{\alpha-1}{\alpha}+\frac{M_{1}}{M_{0}} R=\frac{\alpha-1}{\alpha}+\frac{(\alpha-1)(2 \alpha-5)}{\alpha(3-\alpha)} R .
$$

Она получается при линеаризации по $\dot{h}$ уравнения (16), в котором опущен вклад первого члена (суммы), дающий поправку более высокого порядка $O\left(g^{2}\right)$.
Такое положение линии неустойчивости подтверждает полезность указанной выше физической интерпретации.

Пересечение линии (25) в сторону бо́льших значений $\alpha$ приводит к появлению устойчивого цикла. Непосредственно вблизи нее возникают маленькие овальные циклы, а колебания скорости $\dot{h}(t)$ близки к гармоническим, см. рис. 1.

При углублении в зону неустойчивости, циклы быстро теряют овальную форму, см. рис. 2, и осцилляции приобретают ярко выраженный релаксационный характер, см. рис. 3.

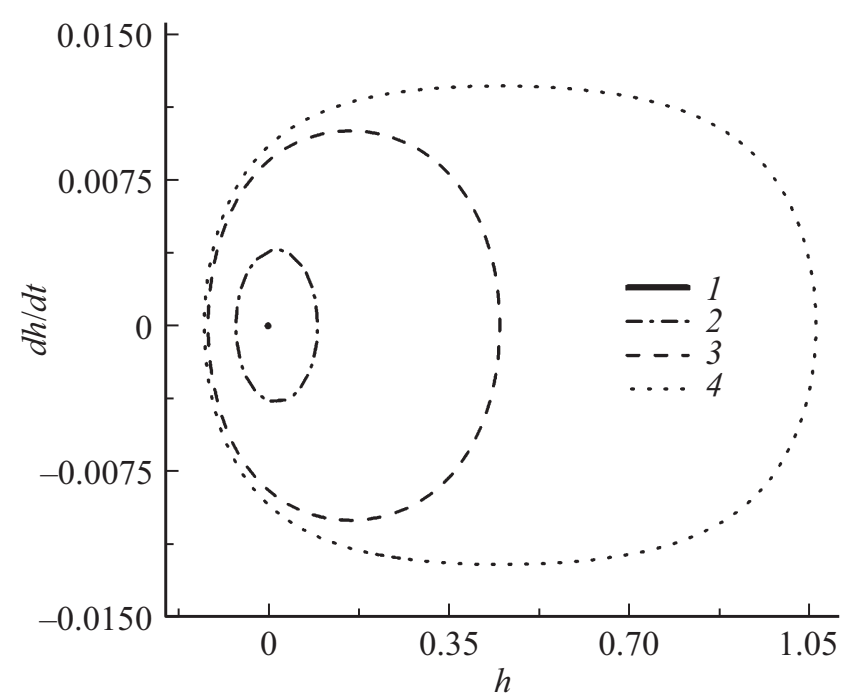

Рис. 1. Устойчивые овальные циклы в ближайшей окрестности линии неустойчивости (25). Значения параметров $R=10^{-4}, \beta=0.04, \ln \left(\frac{V_{L}}{V_{0}}\right)=-3$, линия $1-\alpha=1.041666$, линия $2-\alpha=1.041668$, линия $3-\alpha=1.041675$, линия $4-$ $\alpha=1.0416782$.

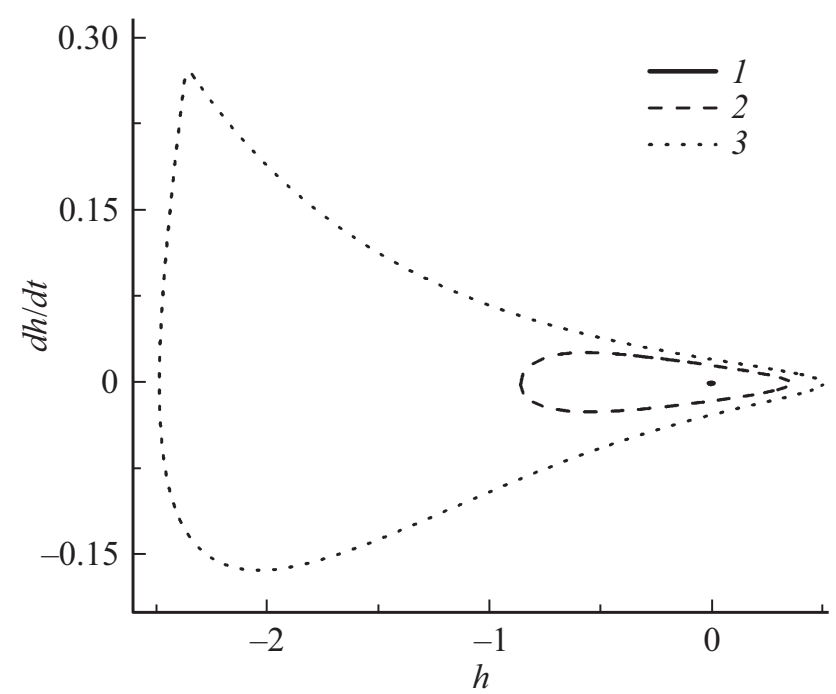

Рис. 2. Циклы квазигармонических (линия 2) и релаксационных колебаний (линия 3 ). Значения параметров $\beta=0.55$, $\ln \left(\frac{V_{L}}{V_{0}}\right)=-20, R=10^{-4}$, линия $1-\alpha=2.222$, линия $2-$ $\alpha=2.223$, линия $3-\alpha=2.2625$. 
При продолжении продвижения вглубь этой зоны возникают два возможных сценария. В одном периодическое движение фронта происходит с его остановка-

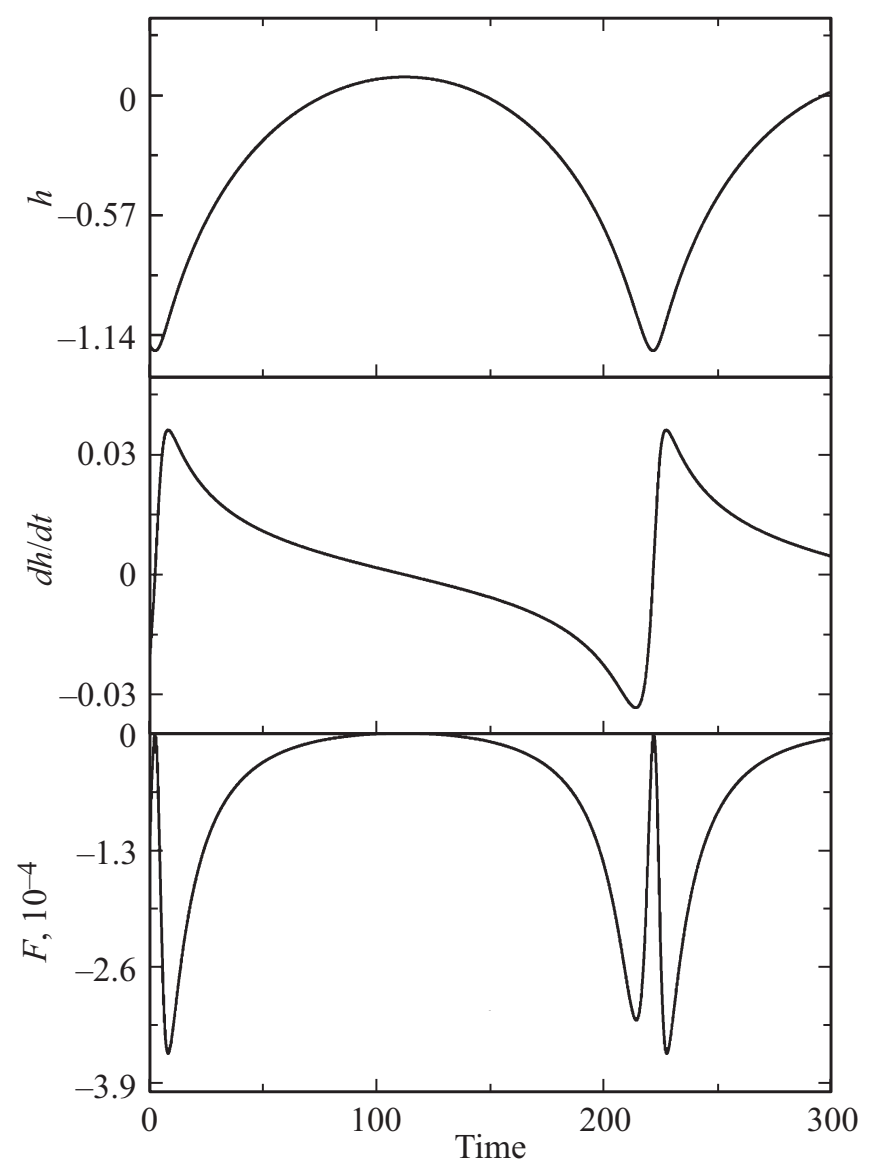

Рис. 3. Режим релаксационных колебаний. Зависимость „вязкой силы“ $F(\dot{h})$ определена соотношением (19). Значения параметров $\beta=0.6, \ln \left(\frac{V_{L}}{V_{0}}\right)=-2, R=10^{-4}, \alpha=2.5006$.

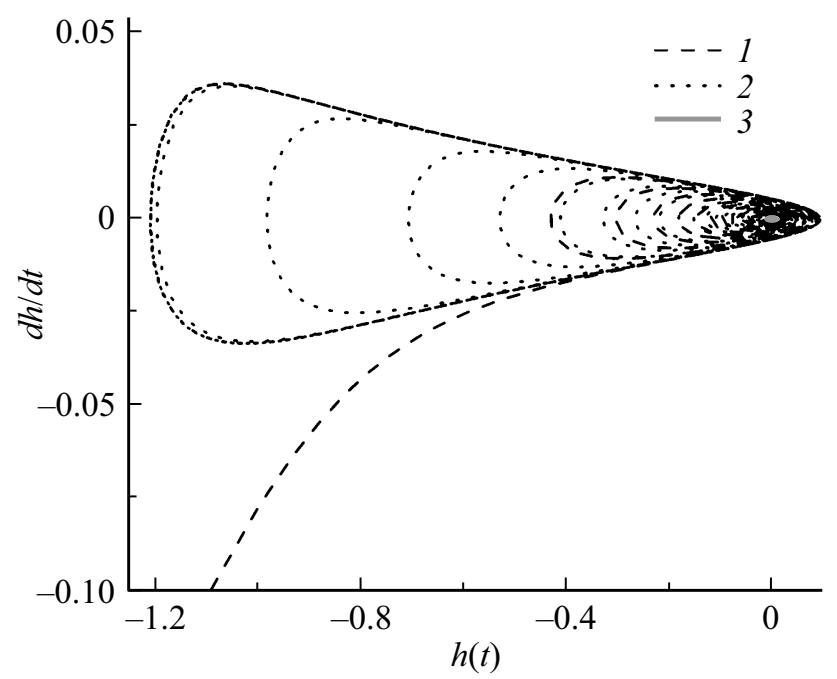

Рис. 4. Остановка фронта ( $h \rightarrow-1$ для линии 1). Значения параметров $\beta=0.6, \ln \left(\frac{V_{L}}{v_{0}}\right)=-2, R=10^{-4}$, линия $1-$ $\alpha=2.5007$, линия $2-\alpha=2.5006$, линия $3-\alpha=2.5$.
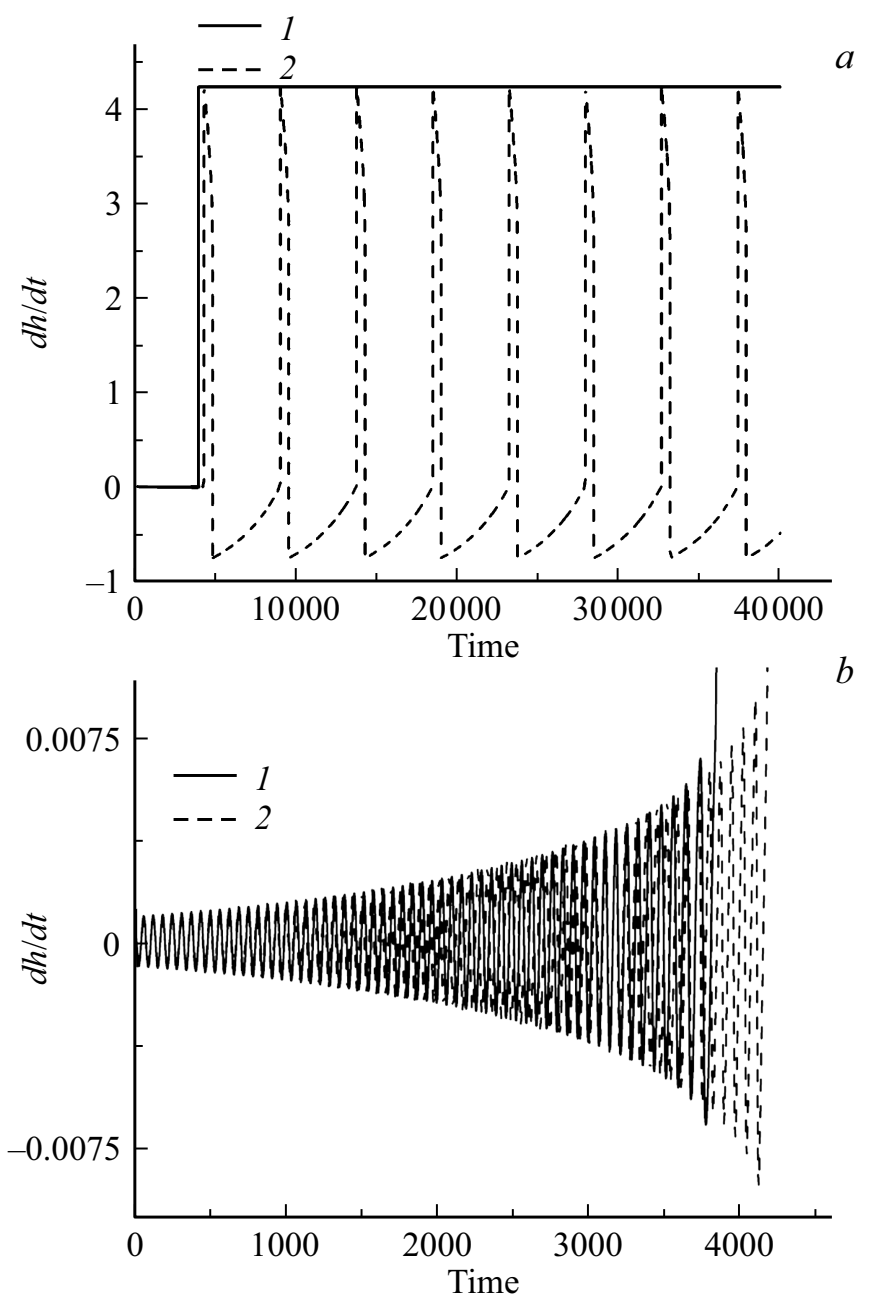

Рис. 5. Иллюстрация важности нелинейности возвращающей силы. Значения параметров $\beta=0.001, R=0.0001$, $\ln \left(\frac{V_{L}}{V_{0}}\right)=-3, \alpha=1.01011$. Линия $1-$ уравнение (22), линия 2 - уравнение (22) с линейной аппроксимацией возвращающей силы. $a$ ) длительный промежуток времени; $b$ ) начальный промежуток времени.

ми. Уравнение (22) позволяет установить факт только первой остановки, см. рис. 4, т.к. вывод уравнения (22) предполагает, что средняя скорость движения равна скорости $V_{L}$, а наличие остановок приводит к более низким средним скоростям.

Другой сценарий связан с существенной нелинейностью возвращающей силы в (22), см. рис. 5 и появлением дополнительного нуля у функции $F(\dot{h})$. Эта ситуация проиллюстрирована рис. 6 , на котором видно, что точка $\dot{h}=0$, отвечающая „лазерной“ моде с $V=V_{L}$ становится неустойчивой $F^{\prime}>0$ (в то время как дополнительный нуль при $\dot{h}>0$ (т.е. с $V>V_{L}$ ) устойчив $\left(F^{\prime}<0\right)$ ) и, следовательно, фронт убегает от лазерного пятна, и ВК переходит в самоподдерживающийся режим.

В таблице приведены значения скорости убегающего фронта, полученные прямым численным решением урав- 
Сравнение нулей функции $F$, заданной формулой (19), и режима убегания уравнения (22)

\begin{tabular}{l|c|c|c}
\hline$\beta_{L}$ & $\alpha=\frac{1.01}{1-\beta_{L}}$ & $\begin{array}{c}\text { Нули } \\
\text { функции } \mathrm{F}(19)\end{array}$ & $\begin{array}{c}\dot{h} \text { в режиме убегания } \\
\text { из решения } \\
\text { уравнения (22) }\end{array}$ \\
\hline 0.02 & 1.03 & 3.04 & 3.03 \\
0.03 & 1.04 & 2.25 & 2.24 \\
0.04 & 1.05 & 1.72 & 1.69 \\
0.05 & 1.06 & 1.33 & 1.29 \\
0.06 & 1.07 & 1.03 & 0.976 \\
0.07 & 1.09 & 0.81 & 0.863 \\
0.08 & 1.100 & 0.63 & 0.66 \\
0.09 & 1.11 & 0.49 & 0.49 \\
0.011 & 1.02 & 4.2 & 4.19 \\
0.012 & 1.022 & 4.04 & 4.03 \\
0.013 & 1.023 & 3.89 & 3.88
\end{tabular}

нения (22) (левая колонка) и положения нулей функции $F(\dot{h})$ (правая колонка).

C учетом того, что численное решение в некоторых случаях могло еще не выйти на асимптотику, можно считать установленным, что скорость самоподдерживающегося фронта $V_{s}=1+\dot{h}$ задается нулем $F(\dot{h})$.

Наши численные расчеты также показывают, что при одних и тех же значениях параметров задачи, но разном выборе начальных значений $h(t=0), \dot{h}(t=0)$, возможна смена динамического режима. В связи с этим отметим, что начальное значение скорости фронта можно задавать, начиная эксперимент с подачи короткого лазерного импульса с $I_{2}=f \delta(t)$, который приведет к скачку скорости $V_{0} \approx \frac{f}{M_{0}} \Delta t$ при условии, что продолжительность импульса $\Delta t \gg \frac{M_{1}}{M_{0}}$. Таким образом можно управлять

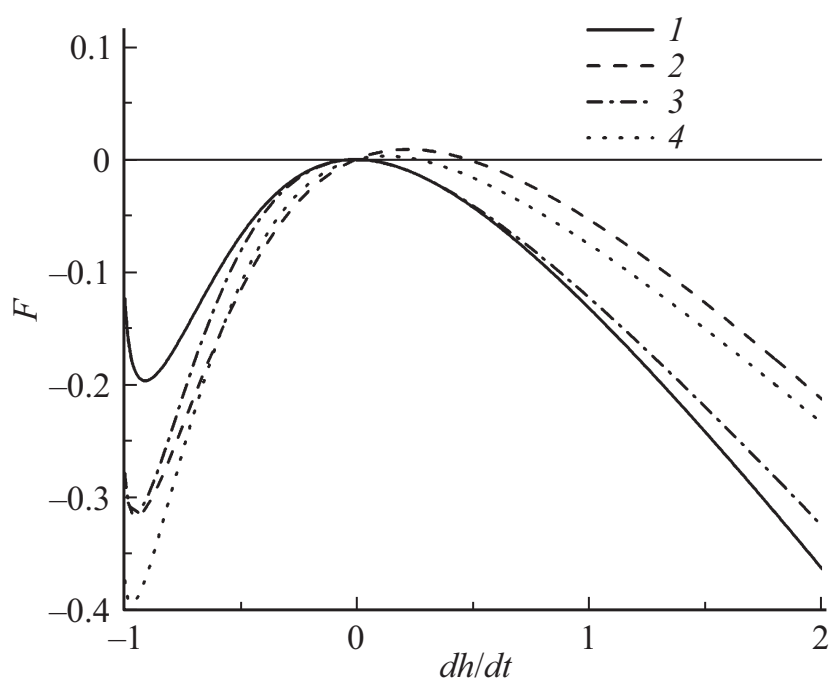

Рис. 6. Зависимость (19). Значения параметров $\ln \left(\frac{V_{L}}{V_{0}}\right)=-3$, линия $1-\beta=0.55, \alpha=2.22$, линия $2-\beta=0.55, \alpha=3$, линия $3-\beta=0.65, \alpha=2.85$, линия $4-\beta=0.65, \alpha=3.5$. начальным выбором режима или переключать его в ходе процесса ВК.

\section{5. Заключение}

В работе предложен аналитический метод расчета динамики фронта ВК с помощью решения выведенного нами приближенного нелинейного дифференциального уравнения. Метод применим в существенно более широкой области параметров по сравнению с имеющимися в литературе теоретическими результатами. Так, результаты работы [17] применимы только в окрестности изолированной точки с координатами $\beta=2 / 3, \alpha=3$. В работе [20] дифференциальное уравнение, описывающее динамику фронта, было выведено только для случая малых амплитуд колебаний скорости, в то время как предложенный нами метод свободен от этого ограничения. Кроме того, в нашей работе численно подробно изучены особенности перехода автоколебательной моды движения фронта в режим его самораспространения с постоянной скоростью. Поскольку метод позволяет выявить общие параметрические зависимости режимов ВК, его результаты являются полезным ориентиром для исследований, целью которых является более детальное описание ВК на мезо- и микроскопических масштабах. Отметим, что использованный в работе подход допускает учет разнообразных лазерных режимов. За счет включения ланжевеновских сил в выведенное уравнение движения межфазного фронта этот поход также может быть применен для описания моды ВК, при которой ведущую роль играет процесс зародышеобразования.

\section{Финансирование работы}

Работа выполнена при финансовой поддержке Российского научного фонда (проект N 19-19-00552).

\section{Конфликт интересов}

Авторы заявляют, что у них нет конфликта интересов.

\section{Список литературы}

[1] И. Пригожин, Д. Кондепуди. Современная термодинамика. От тепловых двигателей до диссипативных структур. Мир, М. (2002). 461 с.

[2] A.S. Rogachev, S.G. Vadchenko, A.S. Aronin, A.S. Shchukin, D.Yu. Kovalev, A.A. Nepapushev, S. Rouvimov, A.S. Mukasyan. J. Alloys Compd. 749, 44 (2018).

[3] V.G. Myagkov, A.A. Ivanenko, L.E. Bykova, V.S. Zhigalov, M.N. Volochaev, D.A. Velikanov, A.A. Matsynin, G.N. Bondarenko. Sci. Rep. 10, 1, 1 (2020).

[4] G.H. Gilmer, H.J. Leamy. In: Laser and Electron-Beam Processing of Materials/Eds C.W. White, P.S. Peercy. Academic, N. Y. (1980). P. 227.

[5] В.А. Шкловский. ЖЭТФ 82, 2, 536 (1982).

[6] W. van Saarloos, J.D. Weeks. Phys. Rev. Lett. 51, 1046 (1983).

[7] В.А. Шкловский, В.М. Кузьменко. УФН 157, 311 (1989). 
[8] А.В. Коропов, В.А. Шкловский. Хим. физика 7, 338 (1988).

[9] G. Auvert, D. Bensahel, A. Perio, T. Nguyen, G.A. Rozgonyi. Appl. Phys. Lett. 39, 724 (1981).

[10] H.J. Zeiger, John C.C. Fan, B.J. Palm, R.I. Chapman, R.P. Gale. Phys. Rev. B 25, 4002 (1982).

[11] D. Bensahel, G. Auvert, A. Perio, J.C. Pfister. J. Appl. Phys. 54, 3485 (1983).

[12] H.-D. Geiler, E. Glaser, Goetz, M. Wagner. J. Appl. Phys. 59, 3091 (1986).

[13] C. Grigoropoulos, M. Rogers, S.H. Ko, A.A. Golovin, B.J. Matkowsky. Phys. Rev. B 73, 184125 (2006).

[14] B.C. Johnson, P. Gortmaker, J.C. McCallum. Phys. Rev. B 77, 214109 (2008).

[15] K. Ohdaira, H. Matsumura. J. Cryst. Growth 362, 149 (2013).

[16] C.E. Wickersham, G. Bajor, J.E. Greene. Solid State Commun. 27, 17 (1978).

[17] W. van Saarloos, J.D. Weeks. Physica D 12, 29 (1984).

[18] D.A. Kurtze, W. van Saarloos, J.D. Weeks. Phys. Rev. B 30, 1398 (1984).

[19] I. Smagin, A. Nepomnyashchy. Physica D 238, 706 (2009).

[20] D.A. Kurtze. Physica D 20, 303 (1986).

[21] D.A. Kurtze. Phys. Rev. B 40, 11104 (1989).

[22] N. Provatas, M. Grant, K.R. Elder. Phys. Rev. B 53, 6263 (1996).

[23] E.J. Albenze, M.O. Thompson, P. Clancy. Ind. Eng. Chem. Res. 45, 5628 (2006).

[24] C. Reina, L. Sandoval, J. Marian. Acta Materialia 77, 335 (2014).

[25] V. Turlo, O. Politano, F. Baras. Acta Materialia 120, 189 (2016).

[26] F. Baras, V. Turlo, O. Politano, S.G. Vadchenko, A.S. Rogachev, A.S. Mukasyan. Adv. Eng. Mater. 20, 8, 1800091 (2018).

[27] S.A. Rogachev, O. Politano, F. Baras, A.S. Rogachev. J. NonCryst. Solids 505, 202 (2019).

[28] L. Nikolova, T. LaGrange, M.J. Stern, J.M. MacLeod, B.W. Reed, H. Ibrahim, G.H. Campbell, F. Rosei, B.J. Siwick. Phys. Rev. B 87, 064105 (2013).

[29] G.C. Egan, T.T. Li, J.D. Roehling, J.T. McKeown, G.H. Campbell. Acta Materialia 143, 13 (2018).

[30] G.C. Egan, T.T. Rahn, A.J. Rise, H.Y. Cheng, S. Raoux, G.H. Campbell, M.K. Santala. J. Appl. Phys. 126, 105110 (2019).

[31] C. Buchner, W. Schneider. J. Appl. Phys. 117, 245301 (2015).

[32] A.L. Korzhenevskii, R. Bausch, R. Schmitz. Phys. Rev. Lett. 108, 046101 (2012).

[33] A.L. Korzhenevskii, R. Bausch, R. Schmitz. Phys. Rev. E 85, 021605 (2012).

[34] А.А. Чеврычкина, Н.М. Бессонов, А.Л. Корженевский. ФTT 61, 11, 2122 (2019).

Редактор Т.Н. Василевская 